Pacific Journal of Mathematics

SOME UNDECIDABILITY RESULTS FOR LATTICES IN 


\title{
SOME UNDECIDABILITY RESULTS FOR LATTICES IN RECURSION THEORY
}

\author{
JEFFREY S. CARROLL ${ }^{1}$
}

\begin{abstract}
A major open question from recursion theory had been whether $\mathscr{E}$, the lattice of recursively enumerable (r.e.) sets, was undecidable. Recently, Harrington and, independently, Herrmann have announced that the lattice is indeed undecidable. Previous to this, Nerode and Smith showed that the lattice of r.e. subspaces of the (canonical) recursive vector space $V_{\infty}$ is undecidable. Their proof involved powerful techniques of recursive algebra. This paper presents two more undecidability results for lattices of r.e. substructures but no advanced recursion theoretic techniques will be required. The primary result of the first section is the undecidability of the lattice of r.e. equivalence relations. Recursive Boolean algebras have been more widely examined and, in the second section, for any infinite recursive Boolean algebra, the lattice of its r.e. subalgebras is shown to be undedicable.
\end{abstract}

\section{The lattice of $r$. e. equivalence relations.}

Definition. Say that $\eta$ is a recursively enumerable (r.e.) equivalence relation if $\eta$ is an equivalence relation over $\mathfrak{R}$ and $\{\langle x, y\rangle \mid x \eta y\}$ is an r.e. set.

We will prefer to think of $\eta$ as a subset of $\mathfrak{R}^{2}$ and the notation “ $(x, y) \in \eta$ " will be used.

R.e. equivalence relations have been used as a tool for recursion theorists, particulary with respect to strong reducibilities (see [Od] for a survey) but it was Ershov [Er] who introduced the lattice of r.e. equivalence relations (therein called "positive equivalences"). The lattice has not been examined as closely as the r.e. substructure lattices of other recursive objects such as vector spaces, Boolean algebras, and fields ([NR1] provides a comprehensive survey of this work). Some recursion theoretic properties for the lattice of r.e. equivalence relations have been studied in [Ca 1,2,3] and the relationship between r.e. equivalence relations and provability in Peano arithmetic has been considered in [Be, BeS, Mo].

We begin this study of the lattice of r.e. equivalence relations by reviewing the basic definitions from [Er] (although we use a different terminology).

\footnotetext{
${ }^{1}$ The author would like to thank Matti Rubin for his help.
} 
If $\eta$ and $\nu$ are both r.e. equivalence relations then $\eta \leq \nu$ (read: $\eta$ is finer than $\nu$; or $\nu$ extends $\eta$ ) if $\eta$ is a finer equivalence relation than $\nu$, that is, if

$$
(\forall x)(\forall y)[(x, y) \in \eta \rightarrow(x, y) \in \nu] .
$$

If $R$ is a r.e. subset of $\Re^{2}$ then $R^{*}$ is the finest r.e. equivalence relation containing $R$ (as a set). Equivalently $R^{*}$ is the closure of $R$ under reflexivity, symmetry and transitivity over $\mathfrak{N}$.

The partial ordering $<$ of the r.e. equivalence relations forms a lattice $\mathscr{P}$ where $\eta \wedge_{p} \nu=_{\mathrm{df}} \eta \cap \nu$ (as sets) and $\eta \vee_{p} \nu=_{\mathrm{df}}(\eta \cup \nu)^{*}$. We define $1_{p}=\mathfrak{N}^{2}$ and $0_{p}=\{(n, n) \mid n \in \mathfrak{N}\}$ (equality).

Contrary to the translation of Ersov [Er], the lattice is not modular. Recall that a lattice $L$ with operations $\Lambda$ and $\vee$ is modular if

$$
(\forall X)(\forall Y<X)(\forall Z)[X \wedge(Y \vee Z)=Y \vee(X \wedge Z)]
$$

We give a counterexample to the modularity. Given a r.e. equivalence relation $\eta$, say that $\eta \equiv\left\langle A_{1}, A_{2}, \cdots\right\rangle$ if each $A_{l}$ is an equivalence class of $\eta$ and $\bigcup_{i} A_{l}=\mathfrak{R}$. We can thus express the r.e. equivalence relations in terms of their equivalence classes. If

$$
\begin{aligned}
& X \equiv\langle\{0,1,4,5, \ldots\},\{2,3,6,7, \ldots\}\rangle, \\
& Y \equiv\langle\{0,1\},\{2,3\},\{4,5\}, \ldots\rangle, \text { and } \\
& Z \equiv\langle\{0\},\{1,2\},\{3,4\}, \ldots\rangle,
\end{aligned}
$$

then $X \wedge(Y \vee Z)=X$, while $Y \vee(X \wedge Z)=Y$.

Ershov's simplest example of a r.e. equivalence relation is $\eta_{R}=_{\mathrm{df}}$ $\{(x, y) \mid x, y \in R\} \cup 0_{p}$, where $R$ is a r.e. set. The mapping that takes the r.e. set $R$ to the r.e. equivalence relation $\eta_{R}$ is a natural one and "nearly" defines a sublattice of $\mathscr{P}$. This relationship between r.e. sets and r.e. equivalence relations will be exploited in obtaining the undecidability of the lattice $\mathscr{P}$.

We shall next consider the atoms of $\mathscr{P}$ and a subclass of coatoms of $\mathscr{P}$. All atoms of $\mathscr{P}$ are the form $A_{\langle i, j\rangle}={ }_{\mathrm{df}}\{(i, j),(j, i)\} \cup 0_{p}$. The coatoms of interest are $C_{f}={ }_{\mathrm{df}}(\mathfrak{R}-\{i\})^{2} \cup\{(i, i)\}$.

Our first goal is to see that the above class of coatoms is definable in $\mathscr{P}$. The definition is due to Burris and Sankappanavar $[\mathbf{B u S}]$ who use it to prove a related result; namely, that the theory of the class of partition lattices on arbitrary sets is recursively inseparable. The proof of the proposition that follows is essentially the first half of their proof. Define $U(x)={ }_{\mathrm{df}} \operatorname{Coatom}(x) \&(\forall y)(\forall z \neq y)[(\operatorname{Atom}(y) \& \operatorname{Atom}(z)$

$$
\left.\left.\&\left(x \vee y=1_{p}=x \vee z\right)\right) \rightarrow \operatorname{Config}(y \vee z)\right]
$$


where $\operatorname{Atom}(x)$ and $\operatorname{Coatom}(x)$ have the obvious definitions and $\operatorname{Config}(x)$ says that the lattice restricted to elements less than or equal to $x$ is isomorphic to the lattice below.

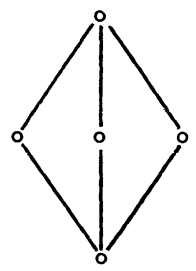

Proposition 1. $\mathscr{P} \vDash U[x]$ iff $(\exists i)\left[x=C_{i}\right]$.

Proof. First consider the coatom $C_{n}$. Let $A_{1}$ and $A_{2}$ be distinct atoms such that $\left(C_{n} \vee A_{1}\right)=1_{p}=\left(C_{n} \vee A_{2}\right)$. Since $\left(A_{i} \vee C_{n}\right)=1_{p}, A_{1}=A_{\left\langle n, J_{1}\right\rangle}$ and $A_{2}=A_{\left\langle n, j_{2}\right\rangle}$ for some $j_{1}$ and $j_{2}$. Consequently $\left(A_{1} \vee A_{2}\right)=$ $\left(\left\{n, j_{1}, j_{2}\right\}\right)^{2} \cup 0_{p}$ which clearly satisfies Config( ).

On the other hand, let $\mathscr{P} \vDash U[E]$ and suppose that $(\forall n)\left[E \neq C_{n}\right]$. Since $E$ is a coatom, $E \equiv\langle J, K\rangle$ where $J \cup K=\mathfrak{R}$ and $J \cap K=\varnothing$. Because $(\forall n)\left[E \neq C_{n}\right],|J|,|K| \geq 2$. Let $j_{1}, j_{2} \in J, k_{1}, k_{2} \in K$ and consider the atoms $A_{1}=A_{\left\langle J_{1}, k_{1}\right\rangle}$ and $A_{2}=A_{\left\langle j_{2}, k_{2}\right\rangle}$. Clearly $A_{i} \vee E=1_{p}$ but $\mathscr{P} \not \equiv$ Config $\left[A_{1} \vee A_{2}\right]$, a contradiction.

Before we begin to show that the lattice $\mathscr{P}$ is undecidable, we shall consider automorphisms of the lattice. Lachlan showed that the lattice of r.e. sets has continuum many automorphisms. Guichard [Gu] has determined that there are only countably many automorphisms of the lattice of r.e. subspaces of the recursive vector space $V_{\infty}$. The result here is attacked in a manner similar to [Gu]. We first need to prove the following proposition.

Proposition 2. Let $\Sigma$ be an automorphism of the lattice of all equivalence relations over $\mathfrak{R} ; \Sigma$ is induced by a permutation on $\mathfrak{R}$.

Proof. First notice that the above definitions and Proposition 1 do not depend on any effective argument; most importantly, $U(x)$ defines the same class of coatoms in the (complete) lattice of all equivalence relations on $\mathfrak{R}$. Also, if $\Sigma(x)=y$ and $U(x)$ holds then $U(y)$ also holds. Given $\Sigma$, we can define a permutation $\pi$ on $\mathfrak{R}$ as follows: if $\Sigma\left(C_{n}\right)=C_{e}$ then $\pi(n)=_{\mathrm{df}} e$. Since $\Sigma$ is an automorphism, $\pi$ is clearly a permutation. It suffices to see that $\pi$ induces $\Sigma$. Define $\Pi(E)={ }_{\mathrm{df}}\{(\pi(x), \pi(y)) \mid(x, y)$ $\in E\}$. 
Clearly $\Pi$ is an automorphism of the lattice and by construction, $(\forall n)\left[\Pi\left(C_{n}\right)=\Sigma\left(C_{n}\right)\right]$. For any atom $A_{\langle i, j\rangle}, A_{\langle l, j\rangle}=\wedge\left\{C_{n} \mid n \neq i, j\right\}$ and so $\Pi\left(A_{\langle i, j\rangle}\right)=\Sigma\left(A_{\langle i, j\rangle}\right)$. Finally, we note that any equivalence relation is a join of atoms, namely $E=\bigvee\left\{A_{\langle i, j\rangle} \mid(i, j) \in E\right\}$, and since an infinite join of atoms is preserved under automorphism, $\Pi(E)=\Sigma(E)$ for any E.

TheOREM 3. $\Sigma \in \operatorname{Aut}(\mathscr{P})$ if and only if $\Sigma$ is induced by a recursive permutation on $\mathfrak{R}$.

Proof. The sufficiency is trivial. To prove the necessity we first note that an automorphism $\Sigma$ of $\mathscr{P}$ can be extended to an automorphism of all equivalence relations and by Proposition 2 , this extension (and so also $\Sigma$ ) is induced by a permutation $\pi$ of $\mathfrak{R}$.

It suffices to show that $\pi$ is a recursive permutation. Let $\pi(n)={ }_{\mathrm{df}} a_{n}$. We shall define two r.e. equivalence relations $E_{1}$ and $E_{2}$ such that with $a_{0}$ we can effectively find $a_{n}$ for any $n$. Define $\eta_{1}$ and $\eta_{2}$ with equivalence class structures as follows:

$$
\begin{aligned}
& \eta_{1} \equiv\langle\{0,1\},\{2,3\},\{4,5\},\{6,7\}, \ldots\rangle ; \\
& \left.\eta_{2} \equiv\langle\{0\},\{1,2\},\{3,4\},\{5,6\}, \ldots\rangle\right) .
\end{aligned}
$$

Both $\eta_{1}$ and $\eta_{2}$ are recursive equivalence relations; let $E_{1}=\Sigma\left(\eta_{1}\right)$ and $E_{2}=\Sigma\left(\eta_{2}\right)$. We claim that given $E_{1}, E_{2}$ and $a_{0}$, we can effectively construct $a_{1}, a_{2}, \ldots$ (hence $\pi$ is recursive).

To find $a_{1}$ : search for and find $y \neq a_{0}$ such that $\left(a_{0}, y\right) \in E_{1}$. Since $E_{1}$ and $\eta_{1}$ have the same equivalence class structure, a unique such $y$ exists, namely $y=a_{1}$.

Assume that $a_{2 k}$ is known and find $a_{2 k+1}$ as follows: search for and find $y \neq a_{2 k}$ such that $\left(a_{2 k}, y\right) \in E_{1}$. As above, $y=a_{2 k+1}$. That $y$ such that $y \neq a_{2 k+1}$ and $\left(a_{2 k+1}, y\right) \in E_{2}$ is $a_{2 k+2}$.

This completes the proof of Theorem 3; since the number of recursive permutations is countable, there are countably many automorphisms of $\mathscr{P}$.

We now turn towards the main goal of showing that number theory is definably embeddable in $\mathrm{Th}(\mathscr{P})$. Our approach is similar to that of [Ru]. Define

$$
\begin{aligned}
\psi_{\in}(x, y, z) \equiv\{ & (U(x) \& U(y)) \\
& \&\{x \neq y \rightarrow(\exists w \leq z)[\operatorname{Atom}(w) \&[w \vee(x \wedge y) \neq x] \\
& \&\{[w \vee(x \wedge y) \neq y] \&[w \vee(x \wedge y) \neq x \wedge y]]\}\} .
\end{aligned}
$$


Proposition 4. $\mathscr{P} \vDash \psi_{\in}[x, y, z]$ iff

$$
(\exists i)(\exists j)\left[x=C_{l} \& y=C_{J} \&(i, j) \in z\right] .
$$

Proof. The necessity is easy, for if $x=C_{i}, y=C_{\text {, and }}(i, j) \in z$ then choose $w=A_{\langle\imath, j\rangle} ; w \leq z$ and $w \vee(x \wedge y)$ does not equal $C_{l}, C_{j}$, or $C_{1} \wedge C_{j}$.

Now suppose that $\mathscr{P} \vDash \psi_{\in}[x, y, z]$ : by Proposition $1,(\exists i)(\exists j)[x=$ $C_{i} \& y=C_{j}$ ]. Suppose $x \neq y$ and let $A_{\langle k, m\rangle}$ be an atom below $z$ that works, that is, $\left[A_{\langle k, m\rangle} \vee\left(C_{i} \wedge C_{j}\right)\right] \neq C_{i}, C_{j}$ or $\left(C_{i} \wedge C_{j}\right)$. Since $C_{i} \wedge C_{j}$ $=\left(\left(\Re_{i}-\{i, j\}\right)^{2}\right)^{*}$ and since $\left[A_{\langle k, m\rangle} \vee\left(C_{i} \wedge C_{j}\right)\right] \neq C_{i} \wedge C_{j}$, we know that $(\{k, m\} \cap\{i, j\}) \neq \varnothing$. Assuming that $k=i, A_{\langle k, m\rangle} \vee\left(C_{i} \wedge C_{j}\right)=$ $C_{l}$, unless $j=m$. The other possibilities are analogous and thus $A_{\langle i, j\rangle}=$ $A_{\langle h, m\rangle} \leq z$, implying that $(i, j) \in z$.

We next define a formula, $\operatorname{Set}^{*}(x)$, which determines those r.e. equivalence relations which are images of the natural map from $\mathscr{E}$ into $\mathscr{P}$.

$$
\begin{aligned}
\operatorname{Set}^{*}(x) \equiv{ }_{\mathrm{df}} & (\forall y)(\forall z)(\exists v \neq y)(\exists w \neq z) \\
& {\left[\left\{\psi_{\in}(y, v, x) \& \psi_{\in}(z, w, x)\right\} \rightarrow \psi_{\in}(y, z, x)\right] . }
\end{aligned}
$$

Notice that the definition implies that $x$ has at most one equivalence class of size greater than one. Hence $\mathscr{P} \vDash \operatorname{Set}^{*}(x)$ if and only if $x=\eta_{R}$ for some r.e. set $R$.

At this point, we could demonstrate that $\operatorname{Th}(\mathscr{E})$ is definable in $\operatorname{Th}(\mathscr{P})$ and obtain the undecidability indirectly (knowing that $\mathscr{E}$ is undecidable); however, we will obtain the undecidability directly and obtain the definability of $\operatorname{Th}(\mathscr{E})$ in $\operatorname{Th}(\mathscr{P})$ afterwards.

It is well known that any relation can be encoded by equivalence relations; however, the following proposition demonstrates that r.e. relations on $\mathfrak{R}$ can be definably encoded by equivalence relations of $\mathscr{P}$. The proposition is an effective version of a theorem of Shelah which was communicated to the author by Matti Rubin.

Proposition 5. Any r.e. $n$-ary relation $R$ on $\mathfrak{N}$ can be coded by $2(n+1)$ r.e. equivalence relations with $2(n+1)$ parameters.

Proof. We shall assume that $R$ is a r.e. binary relation on $\Re$. For any r.e. binary relation our "parameters" will be the sets $S_{i}=\{x \mid x \equiv$ $i(\bmod 3)\}$ for $i=0,1,2$, as well as the sets $\bar{S}_{i}$. Actually, our parameters are the r.e. equivalence relations of the form $\eta_{S}$ for the $S$ 's above; we 
abuse notation and say " $x \in S$ " as shorthand for

$$
\text { “ }(\exists y)\left[y \neq x \&(x, y) \in \eta_{S}\right] " .
$$

For each $i=0,1$ or 2 , we shall construct r.e. equivalence relations $E_{i}^{1}$ and $E_{i}^{2}$ over $\Re$ which shall define $R \cap\left(\bar{S}_{i}\right)^{2}$; we will have $(a b) \in R \cap$ $\left(\overline{S_{i}}\right)^{2}$ if and only if

$$
\left\{a, b \in \bar{S}_{i} \&\left(\exists z \in S_{i}\right)\left[(a, z) \in E_{i}^{1} \&(b, z) \in E_{i}^{2}\right]\right\} .
$$

It is easy to see that $R$ is determined by the disjunction of the three formulae. We shall construct $E_{0}^{1}$ and $E_{0}^{2}$ (the other $E_{i}^{J}$ have analogous constructions). Let $\left(x_{1}, y_{1}\right),\left(x_{2}, y_{2}\right), \ldots$ be an effective enumeration of $R \cap\left(\overline{S_{0}}\right)^{2}$ and let $n_{1}, n_{2}, \ldots$ be an effective enumeration of $S_{0}$ without repetition. Let $A_{0}=\varnothing=B_{0}$, define $A_{s+1}=A_{s} \cup\left\{\left(x_{s+1}, n_{s+1}\right)\right\}$ and $B_{s+1}=B_{s} \cup\left\{\left(y_{s+1}, n_{s+1}\right)\right\}$. Let $A=\bigcup_{s} A_{s}, B=\bigcup_{s} B_{s}$ and define $E_{0}^{1}=$ $A^{*}$ and $E_{0}^{2}=B^{*}$. Clearly, $E_{0}^{1}$ and $E_{0}^{2}$ are r.e. equivalence relations. It is also clear that if $(a, b) \in\left(R \cap\left(\overline{S_{0}}\right)^{2}\right)$ then there exists a $z \in S_{0}$ such that $(a, z) \in E_{0}^{1}$ and $(b, z) \in E_{0}^{2}$.

So suppose that $a, b \in \overline{S_{0}}$ and $z \in S_{0}$ are such that $(a, z) \in E_{0}^{1}$ and $(b, z) \in E_{0}^{2}$. Since $z \in S_{0}, z=n_{k}$ for exactly one $k$. Since $a \in \overline{S_{0}}$, $(a, z) \in E_{0}^{1}$ if and only if $(a, z)=\left(a, n_{k}\right) \in A_{k}$ if and only if $a=x_{k}$, where $\left(x_{k}, y_{k}\right) \in R$. Similarly $(b, z) \in E_{0}^{2}$ if and only if $b=y_{k}$ and thus $(a, b) \in R$.

This completes the proof of Proposition 5.

We are now ready to define formulae in $\mathscr{L}\left(\wedge, \vee, 0_{p}, 1_{p}\right)$ which will allow us to represent number theory. Using the proof of Proposition 5, we will be able to define in $\mathscr{P}$ standard models of number theory with parameters in $\mathscr{P}$; from this we will show that we can characterize these parameters so defining such standard models.

By applying Proposition 5 to the recursive binary relation $<$ and to the recursive ternary relations + and $\times$ for the standard model of arithmetic, there is a sequence of r.e. equivalence relations $e_{1}, e_{2}, \ldots, e_{36}$ $={ }_{\mathrm{df}} \varepsilon$ which code $<,+$, and $\times$. Using the proof of Proposition 5, we can obtain formula $\psi_{<}(x, y, \varepsilon), \psi_{+}(x, y, z, \varepsilon)$, and $\psi_{x}(x, y, z, \varepsilon)$ which will, with an appropriate $\varepsilon$, define the relations for the standard model. For example, $\psi_{<}(x, y, \varepsilon)$ is defined to be the formula

$$
\bigvee_{i=0,1,2}\left[\sigma_{i}(x, y, \varepsilon) \&(\exists z) \tau_{i}(x, y, z, \varepsilon)\right]
$$

where $\sigma_{i}(x, y, \varepsilon)$ is

$$
(\exists u \neq x)(\exists v \neq y)\left[\psi_{\in}\left(x, u, \eta_{\bar{S}_{\iota}}\right) \& \psi_{\in}\left(y, v, \eta_{\bar{S}_{\imath}}\right)\right]
$$


and $\tau_{t}(x, y, z, \varepsilon)$ is

$$
(\exists w \neq z)\left[\psi_{\in}\left(z, w, \eta_{S_{l}}\right) \& \psi_{\in}\left(x, z, E_{i}^{0}\right) \& \psi_{\in}\left(y, z, E_{i}^{1}\right)\right] .
$$

If $x=C_{j}$ and $y=C_{k}$ then $\sigma_{i}(x, y, \varepsilon) \&(\exists z) \tau_{i}(x, y, z, \varepsilon)$ says " $j, k \in \bar{S}_{i}$ $\&(\exists n)\left[(j, n) \in E_{i}^{0} \&(k, n) \in E_{i}^{1}\right]$ " which codes linear order restricted to $\bar{S}_{i}$.

For an arbitrary $\varepsilon$ of the correct arity, define $\left\langle\mathscr{P} ; U, \psi_{<}, \psi_{+}, \psi_{\times}, \varepsilon\right\rangle$ to be the model with universe $\{x \mid \mathscr{P} \vDash U[x]\}$ (which equals $\left\{C_{i} \mid i \in \mathfrak{R}\right\}$ ) and with relations $\psi_{<}(,, \varepsilon), \psi_{+}(,,, \varepsilon)$, and $\psi_{\times}(,,, \varepsilon)$.

From the above, the following proposition is now immediate.

Proposition 6. There exists a finite sequence of r.e. equivalence relations $\varepsilon$ such that

$$
\left\langle\mathscr{P} ; U, \psi_{<}, \psi_{+}, \psi_{\times}, \varepsilon\right\rangle \cong\langle\mathfrak{N} ;<,+, \times\rangle .
$$

We have thus obtained a standard model of number theory which is definable in $\mathscr{P}$ with parameters. To show that the theory of arithmetic is embeddable in $\mathscr{P}$ it shall thus suffice to construct a formula $\chi(\varepsilon)$ such that if $\mathscr{P} \vDash \chi[\varepsilon]$ then $\left\langle\mathscr{P} ; U, \psi_{<}, \psi_{+}, \psi_{\times}, \varepsilon\right\rangle \cong\langle\mathfrak{N} ;\langle,+, \times\rangle$.

Supposing $\chi(\varepsilon)$ exists then given $\delta$, a sentence in number theory, we can define

$$
\delta^{*} \equiv(\forall \varepsilon)\left[\chi(\varepsilon) \rightarrow \delta^{\prime}(\varepsilon)\right],
$$

where $\delta^{\prime}(\varepsilon)$ replaces occurrences of $<,+$ and $\times$ by $\psi_{<}(,, \varepsilon), \psi_{+}(,,, \varepsilon)$ and $\psi_{\times}(, \varepsilon)$ respectively and where the quantifiers $(\forall x) \cdots$ and $(\exists x) \cdots$ are replaced by $(\forall x)[U(x) \rightarrow \cdots]$ and $(\exists x)[U(x) \& \cdots]$. It is easily seen that $\delta \in \operatorname{Th}(\langle\mathfrak{N} ;<,+, X\rangle)$ if and only if $\delta^{*} \in \operatorname{Th}(\mathscr{P})$, which would complete the proof.

To obtain the formula $\chi(\varepsilon)$ we must define a few more formulae. Let $\operatorname{Compl}^{*}(x, y)$ be the formula

$$
\operatorname{Set}^{*}(x) \& \operatorname{Set}^{*}(y) \&\left(x \wedge y=0_{p}\right) \&\left(x \vee y=1_{p}\right) .
$$

We see that $\mathscr{P} \vDash \operatorname{Compl}^{*}[x, y]$ if and only if $x=\eta_{R}, y=\eta_{S}, R \cup S$ $=\mathfrak{H}$ and $|R \cap S| \leq 1$ : hence $S$ and $R$ are recursive sets.

Define Fin* $(x)$ to be the formula

$$
\operatorname{Set}^{*}(x) \&(\forall y \leq x)(\exists z)\left[\operatorname{Set}^{*}(y) \rightarrow \operatorname{Compl}^{*}(y, z)\right] .
$$

Since any infinite r.e. set contains an r.e. nonrecursive subset, $\mathscr{P} \vDash$ Fin* $[x]$ iff $x=\eta_{R}$ where $|R|<\omega$. Note that it is only with these last two definitions that effectiveness is used in the undecidability argument.

With these definitions we can define $\chi(\varepsilon)$ to include the conjunction of the standard axioms for arithmetic (e.g., commutivity, associativity and 
distributivity) where addition, multiplication and linear order are defined in terms of the $\psi_{\mathrm{op}}$ 's. For example, the commutivity of addition is:

$$
(\forall x)(\forall y)(\forall z)\left[\psi_{+}(x, y, z, \varepsilon) \leftrightarrow \psi_{+}(y, x, z, \varepsilon)\right] .
$$

The conjunct of $\chi(\varepsilon)$ which requires the definability of finiteness is the following formula which states that $\psi_{<}(,, \varepsilon)$ is the standard well-ordering of $\mathfrak{R}$ :

$$
\begin{aligned}
(\forall y)\left\{U ( y ) \rightarrow ( \exists r ) \left[\operatorname{Fin}^{*}(r) \&\right.\right. & \\
(\forall x)\{U(x) & \left.\left.\left.\rightarrow\left[\psi_{<}(x, y, \varepsilon) \leftrightarrow \psi_{\in}(x, y, r)\right]\right\}\right]\right\} .
\end{aligned}
$$

That is to say, for any $y=C_{l}$ there exists a finite set $F$ such that $F=\left\{j \mid C, \leq_{\varepsilon} C_{i}\right\}$. Note that the $r$ stated to exist equals $\eta_{F}$ (and if $y=C_{0}$ then $r=0_{p}$ will work).

With the above formulae, $\mathscr{P} \vDash \chi[\varepsilon]$ insures us that

$$
\left\langle\mathscr{P} ; U, \psi_{+}, \psi_{\times}, \psi_{<}, \varepsilon\right\rangle
$$

is a standard model of arithmetic. Thus the following theorem has now been demonstrated.

THEOREM 7. $\operatorname{Th}(\langle\Re ;<,+, \times\rangle)$ is definable in $\operatorname{Th}(\mathscr{P})$.

Before concluding this section, we wish to demonstrate the definability of a number of recursive notions for r.e. equivalence relations (which can be found in [Cal, 2]). First, as promised, we prove the following theorem relating the theory of $\mathscr{E}$ to the theory of $\mathscr{P}$.

THEOREM 8. Th( $\mathscr{E})$, the theory of the lattice of r.e. sets, is definable in $\operatorname{Th}(\mathscr{P})$.

Proof. From the above argument we know that $\operatorname{Set}^{*}[x]$ if and only if $x=\eta_{R}$, for $R$ an r.e. set. Define

$$
U_{i}(x) \equiv{ }_{\mathrm{df}}\left\{\operatorname{Set}^{*}(x) \&\left(x \vee C_{i}=1_{p}\right)\right\} .
$$

LEMMA 8.1. $\left\langle\left\{x \mid \mathscr{P} \vDash U_{n}[x]\right\} ; \wedge, \vee, 0_{p}, 1_{p}\right\rangle$ is isomorphic to $\mathscr{E}$ for any $n$.

Proof of Lemma. We first consider the case when $n=0$ : let $R$ be an arbitrary r.e. set, define $R_{0}=\{x+1 \mid x \in R\} \cup\{0\}$ and let $\Phi: R \rightarrow \eta_{R_{0}}$.

We shall show that $\Phi$ is an isomorphism from $\mathscr{E}$ onto

$$
\left\langle\left\{x \mid \mathscr{P} \vDash U_{0}[x]\right\}, \vee, \wedge, 0_{p}, 1_{p}\right\rangle .
$$


Obviously $\Phi$ is one-to-one. If $\mathscr{P} \vDash U_{0}[x]$ then for some r.e. set $S, x=\eta_{S}$ and since $\eta_{S} \vee C_{0}=1_{p}$, we know that $0 \in S$. So $x$ is in the range of $\Phi$, implying that $\Phi$ is onto. Clearly $\Phi(R \cap S)=\Phi(R) \wedge \Phi(S)$. If $R \cap S=$ $\varnothing$ then $R_{0} \cap S_{0}=\{0\}$ and so $\eta_{R_{0}} \vee \eta_{S_{0}}=\eta_{\left(R_{0} \cup S_{0}\right)}$. Thus even if $R \cap S$ $=\varnothing, \Phi(R \cup S)=\Phi(R) \vee \Phi(S)$. Obviously $\Phi(\mathfrak{R})=1_{p}$; since $\varnothing_{0}=$ \{\}$_{0}=\{0\}$ and $\eta_{\{0\}}=0_{p}$, we have that $\phi(\varnothing)=0_{p}$.

In the general case, define

$$
R_{k}=\{x \mid x \in R \& x<k\} \cup\{k\} \cup\{x+1 \mid x \in R \& x \geq k\} ;
$$

let $\Phi_{k}: R \rightarrow \eta_{R_{k}}$ and proceed accordingly. This completes the proof of the lemma.

Thus if $\delta \in \operatorname{Th}(\mathscr{E})$ then let $\delta^{*} \equiv{ }_{\mathrm{df}}(\forall c)\left[U(c) \rightarrow \delta^{\prime}(c)\right]$, where $\delta^{\prime}(c)$ is the formula $\delta$ with $\vee_{\mathscr{E}}, \wedge_{\mathscr{E}}, \Re$ and $\varnothing$ replaced by $\vee_{p}, \wedge_{p}, 1_{p}$ and $0_{p}$ respectively and such that occurrences of $(\forall x) \cdots$ and $(\exists x) \cdots$ are replaced by $(\forall x)\left[U_{c}(x) \rightarrow \cdots\right]$ and $(\exists x)\left[U_{c}(x) \& \cdots\right]$. Clearly, $\delta^{*} \in$ $\operatorname{Th}(\mathscr{P})$ and so the theorem is proved.

It is also easy to see that there exists a formula Fin $(x)$ such that $\mathscr{P} \vDash \operatorname{Fin}[x]$ iff $x$ is a finite join of atoms of $\mathscr{P}$ :

$\operatorname{Fin}(x) \equiv_{\mathrm{df}}(\exists y)\left[\operatorname{Fin} *(y) \&(\forall z)(\forall w \neq z)\left[\psi_{\in}(z, w, x) \rightarrow \psi_{\in}(z, w, y)\right]\right]$.

With this formula the ideal of finite r.e. equivalence relations is now seen to be definable in $\mathscr{P}$; hence the definitions for maximal and simple r.e. equivalence relations (found in [Cal, 2,3] are also definable in $\mathscr{P}$.

The lower semilattice of r.e. equivalence relations without infinite equivalence classes has a role of importance in [Cal, 2,3], since much of these papers are devoted to constructions of simple and maximal r.e. equivalence relations which do not have any infinite equivalence classes. The next formula defines the elements of this semilattice:

$$
(\forall y)\left[\left(\operatorname{Set}^{*}(y) \& \neg \operatorname{Fin}^{*}(y)\right) \rightarrow(y \wedge x) \neq y\right] .
$$

If $\nu$ has no infinite equivalence classes and $R$ is any infinite r.e. set then $\left(\nu \wedge \eta_{R}\right) \neq \eta_{R}$. On the other hand, if $\nu$ has an infinite equivalence class then let $C$ be one such, $C$ is r.e. and $\left(\nu \wedge \eta_{C}\right)=\eta_{C}$.

2. The undecidability of the lattice of r.e. subalgebras of an infinite recursive Boolean algebra.

Definition. A recursive Boolean algebra $\mathscr{B}$ consists of $|\mathscr{B}|$ which is a recursive subset of $\mathfrak{A}$ and operations $\wedge_{\mathscr{B}}$ (meet), $\vee_{\mathscr{B}}$ (join) and $\neg \mathscr{B}$ (complement) which are partial recursive and under which $|\mathscr{B}|$ becomes a 
Boolean algebra. Remmel [Re] defined this as a "weakly recursively presented Boolean algebra".

If $\mathscr{B}$ is a recursive Boolean algebra then a subalgebra $B$ of $\mathscr{B}$ is a r.e. subalgebra if $|B|$ is a r.e. subset of $|\mathscr{B}|$.

Given a subset $S$ of an arbitrary Boolean algebra $B$, we let $S^{*}$ denote the subalgebra generated by $S$. If $A$ and $C$ are subalgebras of $B$ then by defining $A+C$ as $(A \cup C)^{*}$ and $A \times C$ as $A \cap C$, we form the lattice of subalgebras of $B$, which we denote as $L(B)$. Moreover, if $\mathscr{B}$ is a recursive Boolean algebra then we denote the lattice of r.e. subalgebras by $\mathscr{L}(\mathscr{B})$. Any finite Boolean algebra is completely determined by the cardinality of its atoms; let $B_{n}$ denote the Boolean algebra having $n$ atoms; it is clear that $L\left(B_{n}\right) \cong \mathscr{L}\left(B_{n}\right)$.

Our objective is to show that for any infinite recursive Boolean algebra $\mathscr{B}, \mathscr{L}(\mathscr{B})$ is undecidable. It is worth noting that this result is in contrast with a result of Remmel (see [NR1], Theorem 107) which gives two different representations of a particular Boolean algebra (namely, the Boolean algebra of finite and cofinite subsets of $\mathfrak{R}$ ) such that the lattice of r.e. ideals of one has an undecidable theory while the lattice of r.e. ideals of the other has a decidable theory.

Assume that $\mathscr{B}$ is a recursive Boolean algebra; a subalgebra $B$ is recursive if and only if $|B|$ is a recursive set. We introduce a lattice theoretic condition which guarantees that $B$ is a recursive subalgebra of $\mathscr{B}$.

Definition. Let $B$ and $C$ be subalgebras of $\mathscr{B}$. Say that $B$ is a complement of $C$ if $B \times C=\{0,1\}$ and for all $x \in \mathscr{B}-B,(B+\{x\})^{*}$ $\times C \neq\{0,1\}$. A complement of $C$ is thus a maximal element in the class of subalgebras which do not intersect $C$; a complement need not be unique. Say that $B$ is a strong complement of $C$ if $B$ and $C$ complement one another and $B+C=\mathscr{B}$.

THEOREM 9 (Theorem 3.2 of $[\mathbf{R e}]$ ). Let $\mathscr{B}$ be a recursive Boolean algebra and let $C \in \mathscr{L}(\mathscr{B}) ; C$ is a recursive subalgebra if and only if there is a $B \in \mathscr{L}(\mathscr{B})$ such that $B$ is a strong complement of $C$.

We can thus define the formula

$$
\begin{aligned}
\operatorname{SC}(v) \equiv & (\exists z)[(v \times z=0) \&(v+z=1) \\
& \&(\forall w)\{v<w \rightarrow w \times z \neq 0\} \&(\forall w)\{z<w \rightarrow w \times v \neq 0\}] ;
\end{aligned}
$$

and the formula

$$
\operatorname{Fin}(v) \equiv(\forall z \leq v) \operatorname{SC}(z)
$$


Proposition 10. $\mathscr{L}(\mathscr{B}) \vDash \operatorname{Fin}[x]$ if and only if $x$ is a finite subalgebra of $\mathscr{B}$.

Proof. First notice that $\mathscr{L}(\mathscr{B}) \vDash \mathrm{SC}[x]$ if and only if $x$ has a strong complement in $\mathscr{B}$. From Theorem 9 we know that $\mathscr{L}(\mathscr{B}) \vDash \mathrm{SC}[x]$ if and only if $x$ is a recursive subalgebra of $\mathscr{B}$.

If $x$ is a finite subalgebra of $\mathscr{B}$ then $x$ and all of its subalgebras are recursive and so $\mathscr{L}(\mathscr{B}) \vDash(\forall y \leq x) \mathrm{SC}(y)$. On the other hand, suppose $x$ is an infinite subalgebra of $\mathscr{B}$; if $\mathscr{L}(\mathscr{B}) \vDash \neg \mathrm{SC}[x]$, then we are done. We may then assume that $x$ has a strong complement and hence $x$ is an infinite recursive subalgebra of $\mathscr{B}$. Thus $x$ is also an infinite recursive Boolean algebra, which, by a result of Nerode and Remmel [NR2], must have a r.e. nonrecursive subalgebra $y$. If $y$ had a strong complement $z \in \mathscr{L}(\mathscr{B})$ then $x \times z$ would be a strong complement of $y$ in $\mathscr{L}(x)$. Consequently, $\mathscr{L}(\mathscr{B}) \vDash \neg(\forall y \leq x) \operatorname{SC}(y)$ and the proposition is proved.

Remmel [Re] defines a number of recursive Boolean algebras but we only need to consider his representation for the atomless Boolean algebra. Let $\mathscr{Q}={ }_{\mathrm{df}}\left(\left\{\left[r_{1}, r_{2}\right) \mid r_{1}, r_{2} \in \mathfrak{Q}\right\}\right)^{*}$, that is, elements of $\mathscr{Q}$ are left-closed, right-open intervals over the rationals.

THEOREM 11 (from Theorems 2.2 and 2.5 of [Re]). If $\mathscr{B}$ is an infinite recursive Boolean algebra and $B$ is a finite Boolean algebra then $B \in \mathscr{L}(\mathscr{B})$.

Sketch. If $\mathscr{B}$ has infinitely many atoms then the result is obvious. However if $\mathscr{B}$ has only finitely many atoms then Remmel showed that $\mathscr{B} \cong \mathscr{Q} \times B_{n}$, for some $n$. It suffices to see that $B_{k} \in \mathscr{L}(\mathscr{Q})$ for any $k$. By letting $s_{l}={ }_{\mathrm{df}}[i-1, i) \in \mathscr{Q}$, then it is easy to see that $B_{k} \simeq$ $\left(\left\{s_{1}, s_{2}, \ldots, s_{k}\right\}\right)^{*}$.

We now turn our attention to relevant undecidability results.

Let $T$ be a theory in a language $L$ and let $T_{f}$ be the set of those sentences in $L$ which are finitely refutable in $T$ (a sentence $\psi$ is finitely refutable in $T$ if $\psi$ is false in some finite model of $T$ ). A theory $T$ is recursively inseparable if the sets $T$ and $T_{f}$ are recursively inseparable; that is, there is no recursive set $R$ of sentences in $L$ such that $T \subseteq R$ and $T_{f} \cap R$ is empty.

Burris and Sankappanavar [BuS] showed that the theory of the class of partition lattices over arbitrary sets is recursively inseparable; they accomplish this by defining in it the theory of two equivalence relations. 
The proof consists of two claims: the first claim is the same as Proposition 1 ; the second claim, in which arbitrary pairs of equivalence relations are defined, is similar in flavor to Proposition 4. However, our present interest is in their corollary which follows.

TheOREM 12 [BuS]. Let $\mathscr{K}=\{L(B) \mid B$ is a Boolean algebra $\}: \operatorname{Th}(\mathscr{K})$ is recursively inseparable.

This theorem follows from a succession of corollaries in [BuS] but it can be proved more directly using a theorem of Sachs [Sa]. Sachs showed that the partition lattice of the Stone space of a Boolean algebra $B$ is dually isomorphic to $L(B)$; using standard methods (see [BuS] or [Ra]), Theorem 12 can be derived from the main result of Burris and Sankappanavar by using this isomorphism.

Finally, before we prove the undecidability, define the sentence $\psi_{x}$ from the sentence $\psi$ in the language of lattices by restricting all quantifiers occuring in $\psi$ to elements less than or equal to $x$ (we may assume that $x$ does not occur in $\psi)$. It is not hard to see that the following is true: for all finite $x \in \mathscr{L}(\mathscr{B}), \mathscr{L}(x) \cong \mathscr{L}(\mathscr{B}) \uparrow x$; moreover, if $\mathscr{L}(x) \vDash \psi$ then $\mathscr{L}(\mathscr{B}) \vDash \psi_{x}$. This is true because the subalgebras of the finite algebra $x$ are independent of the Boolean algebra $\mathscr{B}$ of which $x$ is a subalgebra.

THEOREM 13. If $\mathscr{B}$ is an infinite recursive Boolean algebra then $\mathscr{L}(\mathscr{B})$ is undecidable.

Proof. Let $T$ be the theory of subalgebra lattices of Boolean algebras and let $T_{f}$ be the set of finitely refutable sentences in $T$. By Theorem 12 there is no recursive set of sentences which separates $T$ and $T_{f}$.

If we assume that $\mathscr{L}(\mathscr{B})$ is decidable then the set $C={ }_{\mathrm{df}}\{\psi \mid \mathscr{L}(\mathscr{B}) \vDash$ $\left.(\forall v)\left[\operatorname{Fin}(v) \rightarrow \psi_{v}\right]\right\}$ is a recursive set of formulas. The theorem follows by showing that the set $C$ separates $T$ and $T_{f}$.

LEMMA 13.1. $T \subseteq C$.

Proof of lemma. Let $\psi \in T$ and let $x \in \mathscr{L}(\mathscr{B})$. If $\mathscr{L}(\mathscr{B}) \vDash \operatorname{Fin}(x)$ then $x$ is a finite subalgebra by Proposition 10. Since $x$ is a finite Boolean algebra and by the definition of $T, L(x) \vDash \psi$. Since $L(x) \cong \mathscr{L}(x) \cong$ $\mathscr{L}(\mathscr{B}) \uparrow x \vDash \psi, \mathscr{L}(\mathscr{B}) \vDash \psi_{x}$. Thus for any $x \in \mathscr{L}(\mathscr{B}), \mathscr{L}(\mathscr{B}) \vDash\{\operatorname{Fin}(x)$ $\left.\rightarrow \psi_{x}\right\}$ and so $\psi \in C$. 
LEMMA 13.2. $T_{f} \cap C$ is empty.

Proof of Lemma. Let $\psi \in T_{f}$; by definition of $T_{f}$, there is an $n$ such that $\mathscr{L}\left(B_{n}\right)=L\left(B_{n}\right) \vDash \neg \psi$. By Theorem 11, there exists a subalgebra $x_{n} \in \mathscr{L}(\mathscr{B})$ such that $x_{n} \cong B_{n}$. Thus $\mathscr{L}\left(x_{n}\right) \cong \mathscr{L}\left(B_{n}\right) \vDash \neg \psi$; now since $\mathscr{L}\left(x_{n}\right) \cong \mathscr{L}(\mathscr{B}) \uparrow x_{n}, \quad \mathscr{L}(\mathscr{B}) \vDash \neg \psi_{x_{n}}$. Thus $\mathscr{L}(\mathscr{B}) \vDash\left\{\operatorname{Fin}\left(x_{n}\right) \& \neg \psi_{x_{n}}\right\}$ implying that $\psi \notin C$.

\section{REFERENCES}

[Be] C. Bernardi, On the relation provable equivalence and on partitions in effectively inseparable sets, Studia Logica XL, pp. 29-37.

[BeS] C. Bernardi and A. Sorbi, Classifying positive equivalence relations, J. Symbolic Logic, 48 (1983), 529-538.

[BuS] S. Burris and H. Sankappanavar, Lattice-theoretic decision problems in universal algebra, Algebra Universalis, 5 (1975), 163-177.

[Ca1] J. Carroll, Recursively enumerable equivalence relations, Dissertation, University of Wisconsin (1984).

[Ca2] - Simple and maximal recursively enumerable equivalence relations, preprint.

[Ca3] _ Degrees of r.e. equivalence relations, preprint.

[Er] J. Ersov, Positive equivalences, Algebra and Logic, 10 (1973), 26-41.

[Gu] D. Guichard, Automorphisms and large submodels in effective algebra, Dissertation, University of Wisconsin (1982).

[Mo] F. Montagna, Relatively precomplete numerations and arithmetic, J. Philos. Logic, (to appear).

[NR1] A. Nerode and J. Remmel, A Survey of lattices of r.e. substructures, to appear in Recursion Theory, Proceedings of Symposia of Pure Mathematics, Vol. 42, 1985.

[NR2] _ Generic objects in recursion Theory, to appear in The Proceedings of the Conference on Recursion Theory, Oberwalfach, 1984, Springer-Verlag.

[NS] A. Nerode and R. Smith, The undecidability of the lattice of recursively enumerable subspaces, Proceedings of the Third Brazilian Conference on Mathematical Logic, (Recife, 1979), 245-252.

[Od] P. Odifreddi, Strong reducibilities, Bull. Amer. Math. Soc., 4 (1981), 37-86.

[Ra] M. Rabin, A simple method of undecidability proofs and some applications, Logic Methodology and Philosophy of Science Proc. of the 1964 Int'l. Congress, Bar Hillel, ed. pp. 58-68.

[Re] J. Remmel, Recursively enumerable boolean algebras, Ann. of Math. Logic, 14 (1978), 75-107.

[Ru] M. Rubin, The theory of Boolean algebras with a distinguished subalgebra is undecidable, Ann. Sci. Univ. Clermont \#60, Math., 13 (1976), 129-134.

[Sa] D. Sachs, The lattice of subalgebras of a Boolean algebra, Canad. J. Math., 14 (1962), 451-460.

Received September 14, 1984 and in revised form June 25, 1985. Portions of this paper appeared as part of the author's $\mathrm{Ph}$. D. thesis under the supervision of Terrence Millar at the University of Wisconsin. 



\section{PACIFIC JOURNAL OF MATHEMATICS EDITORS}

\author{
V. S. VARAdarajan (Managing Editor) \\ University of California \\ Los Angeles, CA 90024 \\ Hebert Clemens \\ University of Utah \\ Salt Lake City, UT 84112 \\ Charles R. DePrima \\ California Institute of Technology \\ Pasadena, CA 91125
}

R. FINN

Stanford University

Stanford, CA 94305

HeRManN FLASChKa

University of Arizona

Tucson, AZ 85721

RAMESH A. GANGOlli

University of Washington

Seattle, WA 98195

ROBION KIRBY

University of California

Berkeley, CA 94720

\author{
C. C. MOORE \\ University of California \\ Berkeley, CA 94720 \\ H. SAMELSON \\ Stanford University \\ Stanford, CA 94305 \\ HAROLD STARK \\ University of California, San Diego \\ La Jolla, CA 92093
}

\section{ASSOCIATE EDITORS}

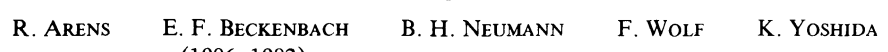

(1906-1982)

\section{SUPPORTING INSTITUTIONS}

\begin{abstract}
UNIVERSITY OF ARIZONA
UNIVERSITY OF BRITISH COLUMBIA

CALIFORNIA INSTITUTE OF TECHNOLOGY

UNIVERSITY OF CALIFORNIA

MONTANA STATE UNIVERSITY

UNIVERSITY OF NEVADA, RENO

NEW MEXICO STATE UNIVERSITY

OREGON STATE UNIVERSITY
\end{abstract}

\author{
UNIVERSITY OF OREGON \\ UNIVERSITY OF SOUTHERN CALIFORNIA \\ STANFORD UNIVERSITY \\ UNIVERSITY OF HAWAII \\ UNIVERSITY OF TOKYO \\ UNIVERSITY OF UTAH \\ WASHINGTON STATE UNIVERSITY \\ UNIVERSITY OF WASHINGTON
}

The Supporting Institutions listed above contribute to the cost of publication of this Journal, but they are not owners or publishers and have no responsibility for its content or policies.

Mathematical papers intended for publication in the Pacific Journal of Mathematics should be in typed form or offset-reproduced (not dittoed), double spaced with large margins. Please do not use built up fractions in the text of the manuscript. However, you may use them in the displayed equations. Underline Greek letters in red, German in green, and script in blue. The first paragraph must be capable of being used separately as a synopsis of the entire paper. In particular it should contain no bibliographic references. Please propose a heading for the odd numbered pages of less than 35 characters. Manuscripts, in triplicate, may be sent to any one of the editors. Please classify according to the scheme of Math. Reviews, Index to Vol. 39. Supply name and address of author to whom proofs should be sent. All other communications should be addressed to the managing editor, or Elaine Barth, University of California, Los Angeles, California 90024.

There are page-charges associated with articles appearing in the Pacific Journal of Mathematics. These charges are expected to be paid by the author's University, Government Agency or Company. If the author or authors do not have access to such Institutional support these charges are waived. Single authors will receive 50 free reprints; joint authors will receive a total of 100 free reprints. Additional copies may be obtained at cost in multiples of 50 .

The Pacific Journal of Mathematics is issued monthly as of January 1966. Regular subscription rate: $\$ 190.00$ a year (5 Vols., 10 issues). Special rate: $\$ 95.00$ a year to individual members of supporting institutions.

Subscriptions, orders for numbers issued in the last three calendar years, and changes of address should be sent to Pacific Journal of Mathematics, P.O. Box 969, Carmel Valley, CA 93924, U.S.A. Old back numbers obtainable from Kraus Periodicals Co., Route 100, Millwood, NY 10546.

The Pacific Journal of Mathematics at P.O. Box 969, Carmel Valley, CA 93924 (ISSN 0030-8730) publishes 5 volumes per year. Application to mail at Second-class postage rates is pending at Carmel Valley, California, and additional mailing offices. Postmaster: Send address changes to Pacific Journal of Mathematics, P.O. Box 969, Carmel Valley, CA 93924.

PUBLISHED BY PACIFIC JOURNAL OF MATHEMATICS, A NON-PROFIT CORPORATION

Copyright $\odot 1986$ by Pacific Journal of Mathematics 


\section{Pacific Journal of Mathematics}

\section{Vol. 122, No. 2 \\ February, 1986}

Gideon Amit and David Chillag, On a question of Feit concerning character values of finite solvable groups ......................257

Constantin Gelu Apostol and Frank Larkin Gilfeather, Isomorphisms modulo the compact operators of nest algebras ................263

Parviz Azimi and James Neil Hagler, Examples of hereditarily $l^{1}$ Banach

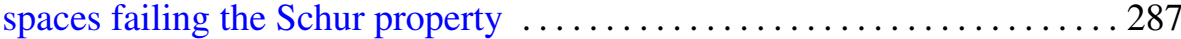

Brian Evan Blank, Boundary behavior of limits of discrete series representations of real rank one semisimple groups . . . . . . . . . . 299

Jeffrey Carroll, Some undecidability results for lattices in recursion theory

Gerald Howard Cliff and Alfred Rheinhold Weiss, Crossed product and

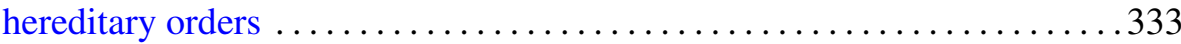

Ralph Cohen, Realizing transfer maps for ramified coverings . . . . . . . . 347

Ronald James Evans, Hermite character sums . .................. 357

C. L. Frenzen and Roderick Sue-Chuen Wong, Asymptotic expansions of the Lebesgue constants for Jacobi series . . . . . . . . . . . . . . . . 391

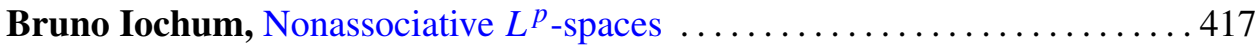

John McDonald, Unimodular approximation in function algebras ....... 435

John Robert Quine, Jr., Ramification and unintegrated value distribution . . 441

Marc Raphael, Commutants of quasisimilar subnormal operators ........ 449

Parameswaran Sankaran and Peter Zvengrowski, On stable

parallelizability of flag manifolds

Helga Schirmer, A relative Nielsen number

Barry Simon, Schrödinger semigroups on the scale of Sobolev spaces . . . . . 475

Viakalathur Shankar Sunder, Stochastic integration in Fock space

Jan de Vries, A note on the $G$-space version of Glicksberg's theorem 